\title{
Archéopages
}

Archéopages

Archéologie et société

\section{Reconnaître et caractériser les zones de forge sur surface décapée. Apport de la géophysique à l'étude paléométallurgique}

Guillaume Hulin, Benjamin Jagou, Marjolaine De Muylder, Alain Henton, Didier Lamotte, Philippe Lefèvre et Aurélie Rousseau

\section{(2) OpenEdition}

Journals

Édition électronique

URL : https://journals.openedition.org/archeopages/560

DOI : $10.4000 /$ archeopages.560

ISSN : 2269-9872

Éditeur

INRAP - Institut national de recherches archéologiques préventives

Édition imprimée

Date de publication : 1 septembre 2014

Pagination : 92-99

ISSN : 1622-8545

Référence électronique

Guillaume Hulin, Benjamin Jagou, Marjolaine De Muylder, Alain Henton, Didier Lamotte, Philippe Lefèvre et Aurélie Rousseau, «Reconnaître et caractériser les zones de forge sur surface décapée. Apport de la géophysique à l'étude paléométallurgique », Archéopages [En ligne], 39 |

10/2013-01/2014, mis en ligne le 01 janvier 2016, consulté le 21 janvier 2022. URL : http:// journals.openedition.org/archeopages/560; DOI : https://doi.org/10.4000/archeopages.560 


\section{Reconnaître et caractériser les zones de forge sur surface décapée Apport de la géophysique à l'étude paléométallurgique}

Guillaume Hulin Inrap, UмR 7619 Metis

Benjamin Jagou Inrap

Avec la collaboration de Marjolaine De Muylder Inrap

Alain Henton Inrap, UMR 8164 Halma-Ipel

Didier Lamotte Inrap, UMR 6298 ARTeHIS

Philippe Lefèvre Inrap

Aurélie Rousseau Inrap

La fouille et l'étude approfondie des sites liés à la métallurgie du fer sont relativement fréquentes. Elles sont généralement menées lorsque l'activité sidérurgique présente des dimensions importantes et que des vestiges caractéristiques sont observables. On pense, par exemple, aux sites de production de fer et à la découverte de restes de bas fourneaux ou de structures de rejet caractéristiques (ferriers). Il en est de même pour les lieux de post-réduction qui ne sont généralement étudiés que lors de la découverte de fortes concentrations de rejets métallurgiques associées à des structures significatives (sol de forge, foyer, fosse de rejet...). La plupart des études se concentrent donc autour de ces grands pôles de production à la signature bien caractéristique. Mais que faire lorsque l'état de conservation du site ne permet pas de mettre en évidence les traces significatives d'une telle activité ? Ou bien tout simplement lorsque ces activités n'ont laissé que peu d'impact sur le sol et que les seuls indices disponibles pour l'archéologue sont la présence de scories en faible quantité découvertes dans le comblement de plusieurs structures non métallurgiques? Ces forges de taille plus modeste et répondant à un besoin ponctuel (réparation d'objets, élaboration d'objets manufacturés en fer...) constituent néanmoins un pan important de l'économie du fer qui ne peut être négligé. Malgré cela, force est de constater que les études sur ce type d'espace, pourtant très courant, représentent un des parents pauvres de la recherche archéologique actuelle. Le recours à certaines techniques géophysiques peut permettre de pallier partiellement ce problème. 


\section{Des indices peu visibles}

Le martelage du fer sur l'enclume produit un micro-déchet caractéristique que l'on nomme battiture et qui permet à l'archéologue d'identifier sans ambiguïté un espace comme étant dédié à la forge. Ce type de déchet est issu d'une couche d'oxydation qui se forme lors du passage du métal dans le foyer de forge dès $300{ }^{\circ} \mathrm{C}$ (Mangin, 2004). Lorsque le métal est extrait du foyer, cette couche, au contact de l'air, se refroidit, se fragilise et, lors de la frappe du métal sur l'enclume, se brise en petits éléments millimétriques. Ces derniers vont alors se déposer et se retrouver piégés sur le sol de la forge en périphérie proche de la zone de martelage et ce malgré les séquences de nettoyage qu'il a pu connaitre. La forte concentration de ces microdéchets magnétiques dans les sédiments permet donc de détecter la présence d'une forge sur un site. Comme l'ont démontré les recherches portant sur ce matériel (Leblanc, 2001), il existe plusieurs morphologies de battitures produites par différents procédés techniques avec notamment des battitures lamellaires et des battitures globulaires. Les lamellaires sont de petites écailles plates qui résultent du martelage d'une surface plane. Les globulaires sont quant à elles semblables à des petites billes qui se forment généralement avec l'emploi de silice lors d'un travail de soudure [ill. 1].

Du fait de leur taille, lidentification des épandages de battitures est extrêmement délicate. Généralement, seules les fortes concentrations associées à des couches charbonneuses vont être repérées et potentiellement étudiées. Le plus souvent, le protocole mis en place consiste, soit à implanter un carroyage pour la réalisation de prélèvements lorsqu'un sol de forge est supposé, soit à prélever une partie du sédiment des structures en creux ayant livré des rebuts métallurgiques. Dans ce cas, l'objectif est double, d'une part cerner l'étendue des micro-déchets présents et d'autre part les caractériser. Si cette méthode peut s'avérer efficace sur les ensembles bien circonscrits, il n'en est pas de même sur les zones de forge plus modestes ou mal préservées, entraînant dès lors des difficultés évidentes de lecture.

\section{L'utilisation de la géophysique}

Les techniques géophysiques sont souvent employées pour la détection des vestiges liés au travail du fer, du fait de leurs propriétés magnétiques très marquées, notamment avant fouille (Cabboï, Dunikowski, 1995 ; Vernon et al., 1998 ; Crew, 2002 ; Bonnamour et al., 2009 ; Gustafsson, Viberg, 2012). Dans de rares cas, la géophysique a été utilisée directement sur surface décapée comme sur l'atelier de forge de Rauranum (Dabas et al., 2002) ou bien encore ceux de Beaunela-Rolande (Best, Marmet, à paraître). À noter que pour ces deux sites, l'activité de forge avait déjà été clairement identifiée par la présence de vestiges significatifs (sols de forge en place, foyers...) lors de la fouille.
La démarche présentée ici s'intéresse aux entités n'ayant laissé que très peu d'indices et que la fouille seule n'aurait pas pu déceler. Cette approche a été initiée et mise en place pour la première fois sur la fouille de Saultain « rue Barbusse » (Nord) en octobre 2011 (Henton, à paraître) puis adoptée sur le projet du canal Seine-Nord Europe. Ce projet d'envergure a été l'occasion de mettre en ouvre une étude géophysique quasi systématique après décapage des horizons superficiels (Hulin et al., 2014). Des mesures de susceptibilité magnétique ont ainsi permis de détecter un certain nombre d'anomalies dont quelques-unes se sont révélées être associées à la présence de battitures traduisant clairement l'emplacement de forges. Au total trois sites ont montré de tels indices sur le tracé du canal, à savoir les sites de Noyon (Oise) [cf. encadré p. 95], Éterpigny-Barleux (Somme) [cf. encadré p. 96] et Sauchy-Lestrée (Pas-de-Calais) [cf. encadré p. 98]. Par la mise en relation de ces trois cas, cet article vise à présenter la méthodologie et son application dans différents contextes archéologiques et à insister sur les multiples apports scientifiques et opérationnels d'une telle approche, en archéologie préventive notamment.

\section{La cartographie géophysique}

La première étape du travail consiste en une étude géophysique réalisée sur l'intégralité de la zone de fouille après décapage des horizons superficiels. Cette étude rapide permet d'appréhender le site archéologique en mesurant un paramètre bien spécifique : la susceptibilité magnétique. Cette propriété physique, qui correspond à la capacité d'un corps à s'aimanter sous l'action d'un champ magnétique, est depuis longtemps reconnue comme un marqueur fort de l'anthropisation des sols (Le Borgne, 1955 ; Aitken, 1958 ; Le Borgne, 1965 ; Tite, Mullins, 1971). Pour exemple, les phénomènes de chauffe (incendie de bâtiment, zone foyère, essartage), le taux de matière organique et bien évidemment les activités liées au travail du fer (présence de micro-déchets) sont autant de paramètres pouvant avoir une origine anthropique et qui ont pour point commun de modifier la teneur et la composition des oxydes et hydroxydes de fer dans le sol, modifications qui se traduiront par des changements dans les propriétés magnétiques des sols (Le Borgne, 1955 ; Mullins, 1974; Marmet, 200o). De ce fait, la cartographie de ce paramètre apporte à l'archéologue une caractérisation supplémentaire de son site et certains phénomènes anthropiques peuvent être mis en évidence sans qu'aucun indice visuel ne soit présent.

D'un point de vue technique, l'utilisation de la sonde monospire Bartington $\mathrm{MS} 2 \mathrm{D}$, spécialement adaptée à l'archéologie, a été préconisée pour ces études [ill. 2]. Cet appareil de mesure électromagnétique offre une profondeur d'investigation d'environ $10 \mathrm{~cm}$ avec une décroissance rapide de la réponse sur les premiers centimètres (Benech, Marmet, 

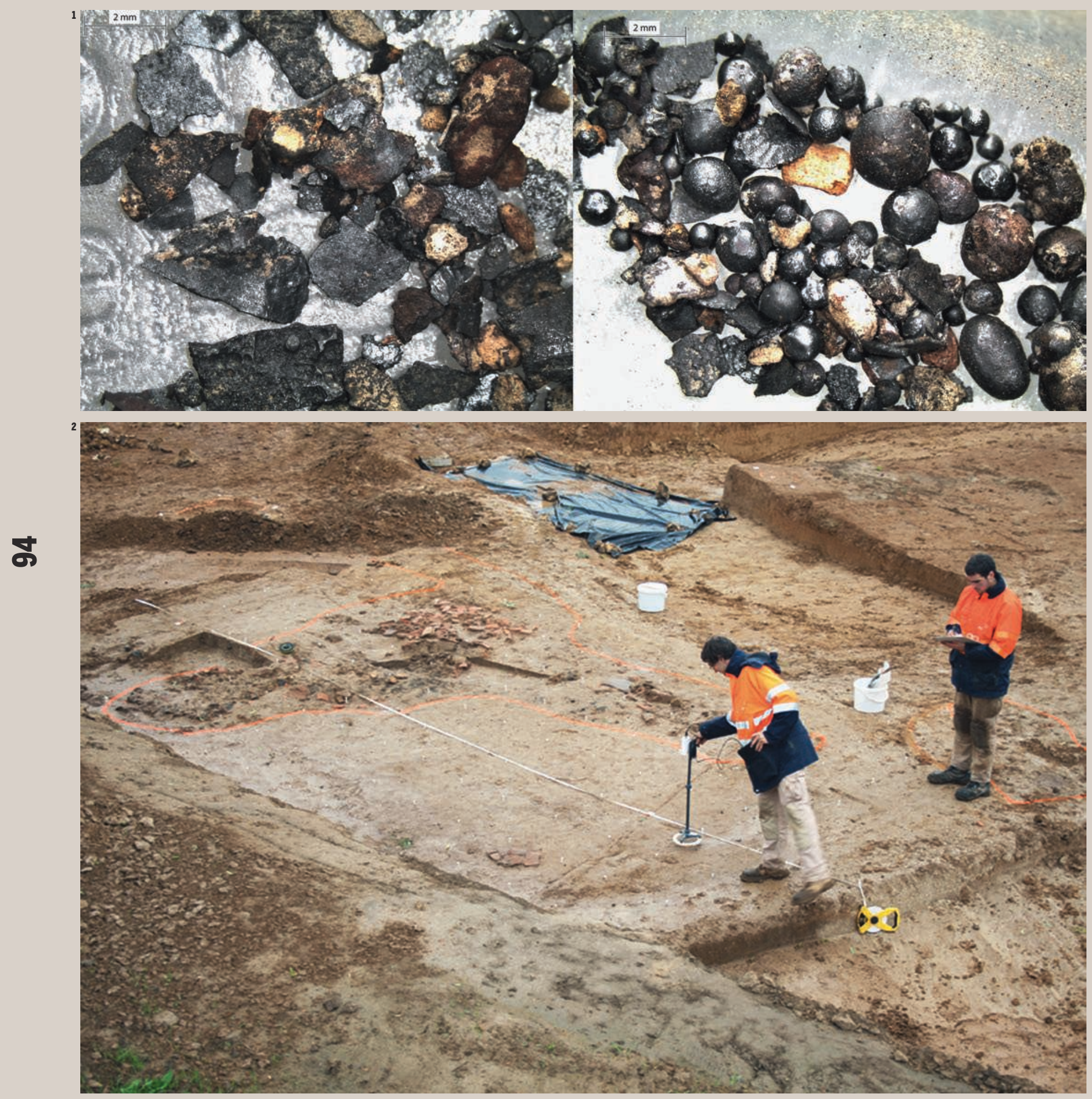

1. Vue sous microscope

de battitures découvertes

sur le site de Noyon (Oise).

Sur la gauche des battitures

lamellaires et sur la droite

des globulaires.

2. Prise de mesure à l'aide

du capteur Bartington MS2B

et mise en place du carroyage

pour les prélèvements

sédimentaires sur le site

de Saultain (Nord). 


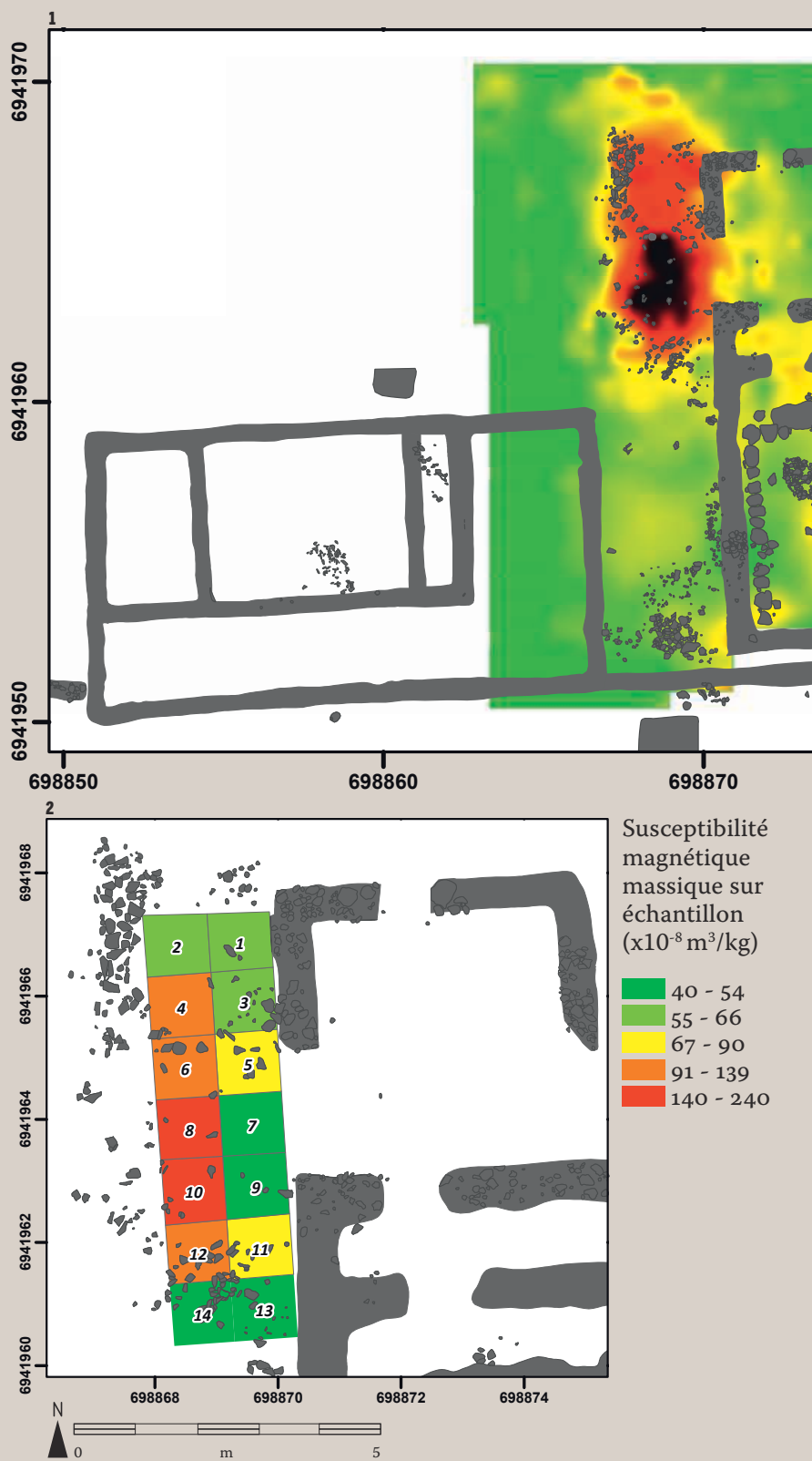

1. Cartographie de

susceptibilité magnétique au-niveau d'une ruelle séparant deux bâtiments

de la villa gallo-romaine de Noyon (coord. Lambert 93).

Susceptibilité

magnétique

volumique $\left(\mathrm{x} 10^{-5} \mathrm{uSI}\right)$
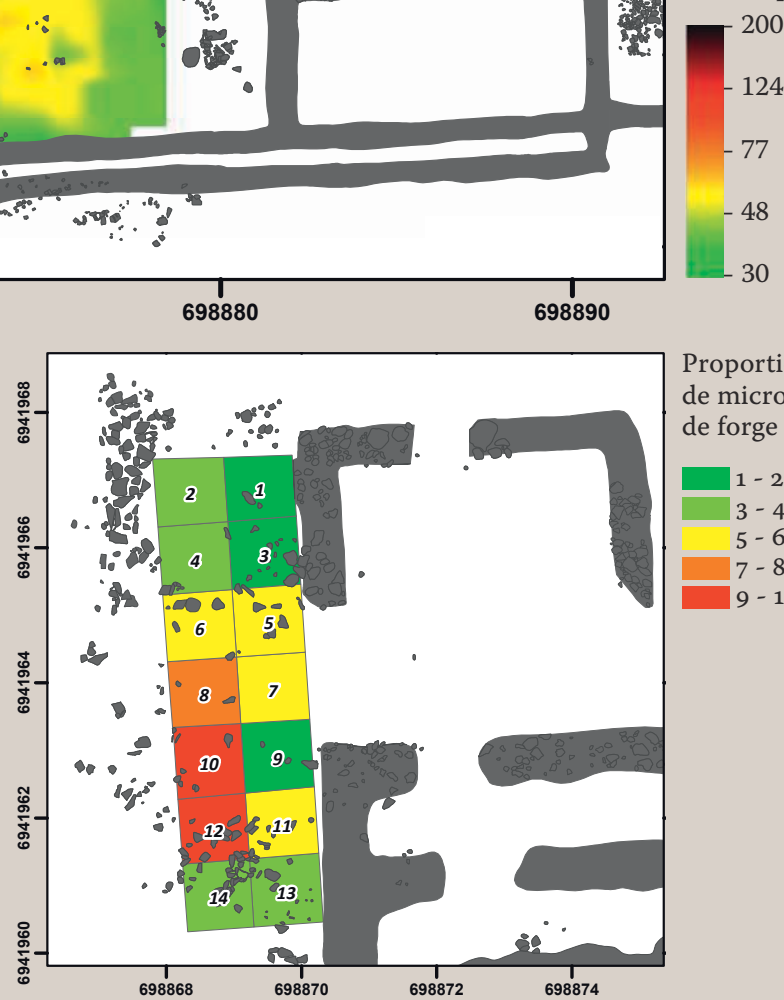

Proportion de micro-déchets de forge (\%)

$1-$

$3-4$

$5-6$

$7-8$

2. Cartographie de susceptibilité magnétique sur échantillons et proportion de micro-déchets de forge au nord-ouest d'un des bâtiments de la villa galloromaine de Noyon. Les deux approches montrent les mêmes tendances générales et notamment un « vide ॥ au niveau du carré 9 pouvant indiquer l'emplacement de l'enclume avec une répartition des battitures autour de la zone de martelage (coord. Lambert 93).
Une forge associée à une villa gallo-romaine à Noyon (Oise)

L'étude géophysique réalisée sur la villa galloromaine de Noyon a mis en évidence une zone anormalement forte en termes de susceptibilité magnétique au nord-ouest d'un des bâtiments de l'aille nord. [ill. 1]. Cette anomalie se distingue par des valeurs particulièrement élevées et possède des limites bien marquées. L'observation de terrain et un test à l'aimant ont rapidement permis d'associer cette anomalie de susceptibilité magnnétique à la présence de battitures. D'un point de vue contextuel, cette anomalie se développe au-dessus du sol construit d'une ruelle située entre deux bâtiments. Ce sol est constitué d'une importante quantité de nummulites et de terres cuites architecturales dont la présence fut particulièrement importante à prendre en compte pour l'étude géophysique et paléométallurgique. Pour cet espace, un carroyage avec une maille d'un mètre a été implanté afin de pouvoir prélever dix litres de sédiments par carré. De par la présence en grande quantité de nummulites et de terres cuites architecturales, les proportions de micro-déchets magnétiques sont relativement faibles. Malgré cela, une première carte de répartition a pu être réalisée [ill. 1], Celle-ci, en corrélation avec les résultats de l'étude géophysique, a offert la possibilitité de cerner un espace où de plus fortes concentrations sont à noter. L'étude sou.s microscope a montré la présence de battitures lamellaires et globulaires dans ce même espace, ce qui permet donc d'y voir le probable lieu où était pratiqué le forgeage du métal. ॠn parallèle et afin de compléter les données de terrain, chaque carré a fait l'objet de mesures de susceptibilité sur échantillons. Les résultats [ill. 2] divergent quelque peu de ceux obtenus directement sur le terrain, mais permettent très clairement de s'affranchir des perturbations liées aux éléments constitutifs du sol puisque les mesures ont été réalisées sur une fraction inférieure à 2 mm éliminant ainsi les nummulites et les fragments de terres cuites. Ils sont donc, en ce sens, particulièrement pertinents et peuvent être comparés avec les données paléométallurgiques. On voit par cet exemple que, sans l'apport de l'étude géophysique, il aurait été difficile de cerner réellement les limites voire connaître l'existence de cette forge datée entre le $\mathrm{Ir}^{\mathrm{e}}$ et le $\mathrm{Iv}^{\mathrm{e}}$ siècle de notre ère (De Muylder et al., à paraître).

\section{ヶ




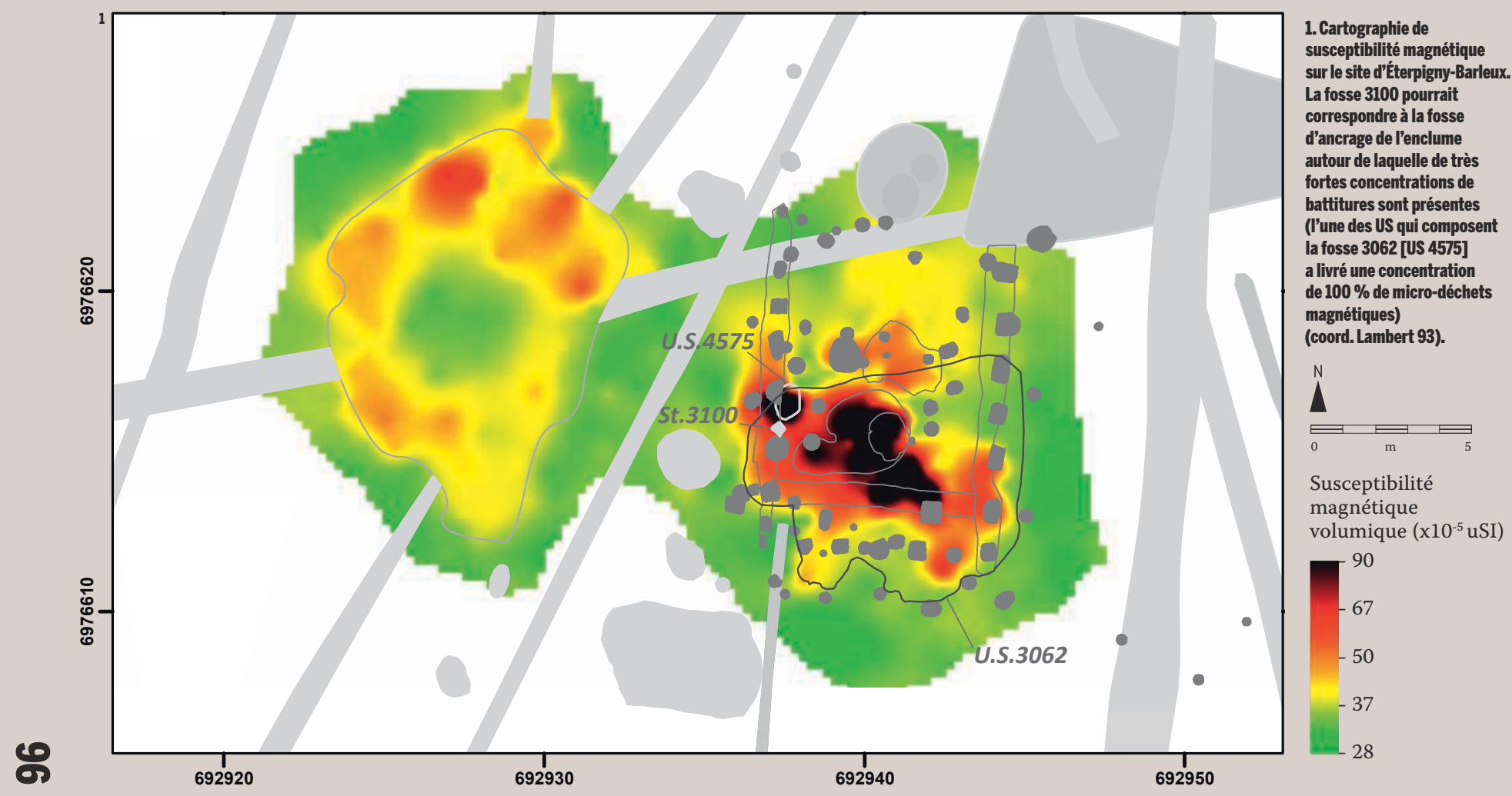

Trois forges successives ( I $^{\mathrm{er}}-\mathrm{II}^{\mathrm{e}}$ siècle de notre ère) à Đterpignny-Barleux (Somme)

D'un point de vue géophysique, plusieurs démarches ont été mises en cuvre sur les différents secteurs d.e fouille de la plate-forme d'J̈terpigny-Barleux ${ }^{1}$. L'une d'elles concernait l'étude des grandes fosses aux fonctions mal définies (fosses d'extraction, mares...) et pour lesquelles la mesure de la susceptibilité peut apporter des éléments de réflexion (Hulin et al., 2014). À la suite du décapage, deux grandes fosses aux contours diffus et comblées d'un sédiment grisâtre sont notamment apparues. Aucun indice n'indiquait la présence d'une forge. Les mesures de susceptibilité maǵnétique réalisées à leur surface ont montré des différences notables entre ces deux fosses aussi bien en termes de valeurs moyennes que de variations internes. Ainsi, l'une d'elles, la fosse 3062 se détache clairement en montrant des valeurs extrêmement fortes. I'observation visuelle et un test à l'aimant ont permis d'associer cette anomalie de susceptibilité magnétique à la présence de battitures [ill. 1]. Une série de prélèvements de deux litres de sédiments a été effectuée sur chaque anomalie magnétique et à ses abords. I'observation des refus de tamis sou.s microscope a confirmé la présence de battitures dans les échantillons des unités stratigraphiques fortement magnnétiques. Une carte de répartition des micro-déchets magnnétiques a ainsi pu être đressée en complément de la carte de susceptibilité magnétique. I'intégration des données issues de la fouille a entraîné la délimitation d'un espace de martelage organisé autour de la structure 3100 , interprétée comme la fosse servant d'ancrage à l'enclume. Une enclume en grrès a par ailleurs été retrouvée en position secondaire dans la grande fosse dépotoir contemporaine de la forge et située quelques mètres plus à l'ouest.

'Trois états d'un bâtiment abritant la forge ont été reconnus par la fouille. Après la destruction des deux premiers, la reconstruction du troisième atelier a largement détruit les structures précédentes, en particulier les niveaux de sol. C'est donc sur les vestiges mieux conservés de la dernière phase que les mesures đe susceptibilité magnétique et les prélèvements d'échantillons ont été réalisés. D'autres méthodes d'investigation (micromorphologie, stratigraphie, etc.) ont cependant été employées afin de comprendre l'architecture et l'évolution des deux premiers bâtiments.

Comme pour le cas de Noyon, la mise en évidence, par la susceptibilité magnnétique, de concentrations de battitures a donné lieu à une localisation plus précise des faits archéologiques. Cette information supplémentaire, disponible rapidement après le décapage, a donné la possibilité d'orienter les recherches et d'adapter les méthodes de fouille. Pour la forge gallo-romaine d'F́terpigny-Barleux, les anomalies détectées par la prospection géophysique ont donc amené les archéologues à pratiquer une fouille manuelle fine, en plan, et effectuer les prélèvements suffisants et nécessaires à l'étude des micro-déchets métalliques. La synthèse des résultats des différentes études a ainsi aidé à la compréhension de ces ateliers de forge utilisés entre le milieu du r ${ }^{\mathrm{er}}$ siècle et le milieu du. $\mathrm{r}^{\mathrm{e}}$ siècle de notre ère (Lamotte et al., à paraître). 
1999). Sur le terrain, la visualisation des faits archéologiques permet d'adapter la collecte de points. Cette prise de mesure sélective s'avère particulièrement importante lorsqu'il convient de ne pas prendre en compte des artefacts naturels évidents (chablis) et autres perturbations anthropiques (vestiges de la Première Guerre mondiale par exemple). Le positionnement des données de susceptibilité est effectué en temps réel grâce à un GPS de précision décimétrique.

La démarche mise en œuvre sur l'ensemble des sites étudiés a été, dans un premier temps, de couvrir la zone d'étude sans enregistrement des données afin d'évaluer rapidement les variations de susceptibilité magnétique et l'intérêt d'une étude plus précise. Ce protocole se base généralement sur la réalisation de transects recoupant les entités archéologiques majeures (bâtiments, enclos fossoyés, grandes fosses...) afin de déceler d'éventuels effets de parois ou zones anormalement fortes. Dans ce cas, un échantillonnage de l'ordre du mètre avec une densification des points de mesures sur les zones d'intérêt est alors effectué pour cartographier précisément l'anomalie. Pour certaines études, les mesures de terrain ont été complétées par des mesures de susceptibilité sur échantillons.

\section{L'étude paléométallurgique}

Une fois repérés les espaces de travail du fer potentiels, un protocole de prélèvement des sédiments est mis en place afin de mieux cerner la présence de micro-déchets magnétiques. La quantité de sédiments collectée varie selon la nature de l'espace testé. Pour les structures en creux, deux litres de sédiments seront prélevés. Pour les possibles sols de forge, un carroyage recouvrant leur superficie globale d'un maillage carré d'un mètre sur un mètre sera implanté. L'état de conservation des sols testés va déterminer dans un premier temps la quantité de sédiments prélevée pour chacun des carrés. Les retours d'expérience ont permis de passer de prélèvements moyens de dix litres à des prélèvements de trois litres par carré. Les échantillons de sédiments sont ensuite pesés, lavés et tamisés par le biais d'une colonne composée de tamis d'ouverture $2 \mathrm{~mm}$ et $500 \mu \mathrm{m}$. Une fois les différents refus de tamis secs, un tri à l'aide d'un aimant est effectué afin de séparer les éléments magnétiques. Les refus magnétiques et ceux non-magnétiques sont ensuite pesés afin de déterminer la proportion d'éléments magnétiques présents dans chacun des prélèvements. Une première carte est ensuite établie en vue de réaliser une répartition de ce type de matériel et ainsi une comparaison avec la cartographie de susceptibilité magnétique. Après analyse des deux approches, un nouvel échantillonnage est effectué au sein des fortes concentrations d'éléments magnétiques et aux endroits de forte corrélation entre les deux études. Ces échantillons, d'environ dix grammes de micro-déchets magnétiques, sont alors observés sous microscope.
L'objectif de ces analyses est de caractériser les différents éléments composant

ces micro-déchets magnétiques et plus particulièrement d'observer la présence ou non de battitures. De cette dernière carte, si l'ensemble des observations s'est avéré positif, il est alors possible de dégager une première interprétation sur l'organisation spatiale de la forge.

\section{La nécessité d'une approche pluridisciplinaire}

Les différentes études présentées dans les encadrés ont pu être menées à bien par la mise en place d'une démarche pluridisciplinaire associant prospection géophysique et étude paléométallurgique. Ces trois cas illustrent l'intérêt d'adopter ce type de protocole lors de la fouille et ce dans différents contextes allant de l'étude sur sol ou structure archéologique à l'étude sur sol naturel. Dans ce dernier cas, il s'agit de déceler des éléments magnétiques ayant migré en profondeur par bioturbation notamment. Cette méthode peut également être utilisée en coupe stratigraphique. L'intérêt est ici particulièrement fort puisque les mesures de susceptibilité vont alors aider à caractériser chacune des unités stratigraphiques identifiées et à indiquer très rapidement la présence de micro-déchets magnétiques particulièrement difficiles à voir en coupe du fait d'une fenêtre d'observation réduite (Hulin et al., 2014). Dans ce cas, le protocole d'étude diffère quelque peu, notamment pour ce qui est de l'échantillonnage et du capteur à utiliser.

Les informations apportées par l'association des données géophysiques et paléométallurgiques sont complémentaires et particulièrement pertinentes : la prospection géophysique permet de cerner rapidement les espaces ayant pu accueillir de possibles ateliers de forge sur les sites archéologiques. Plus précisément, ce type de travail offre la possibilité d'observer des espaces où les traces archéologiques ne sont plus visibles à l'œil « nu », comme sur la fouille de Sauchy-Lestrée, ou lorsque l'état de conservation des sols ne permet pas de bien les identifier, comme sur l'atelier de Noyon. Ces informations révélées par la prospection géophysique, parce qu'elles offrent une lecture directe sur le terrain, permettent d'aborder aussi de manière plus fine la fouille des forges et ainsi de pouvoir collecter toutes les informations spécifiques à ce type d'occupation en aboutissant à une image plus précise de l'organisation de l'atelier (comme pour le site d'Éterpigny-Barleux). Dans ce dernier cas, la fouille des espaces détectés par la prospection a mis en évidence plusieurs bâtiments successifs et leurs structures (foyers, possible emplacement d'enclume, poteaux et solives...). Enfin, une fois ces secteurs bien définis, les observations géophysiques donnent la possibilité de formuler des hypothèses sur l'organisation interne de l'atelier et ainsi de mettre en place un protocole de prélèvements et d'analyses des sédiments mieux ciblé. La fonction première des prélèvements n'est donc plus de délimiter 

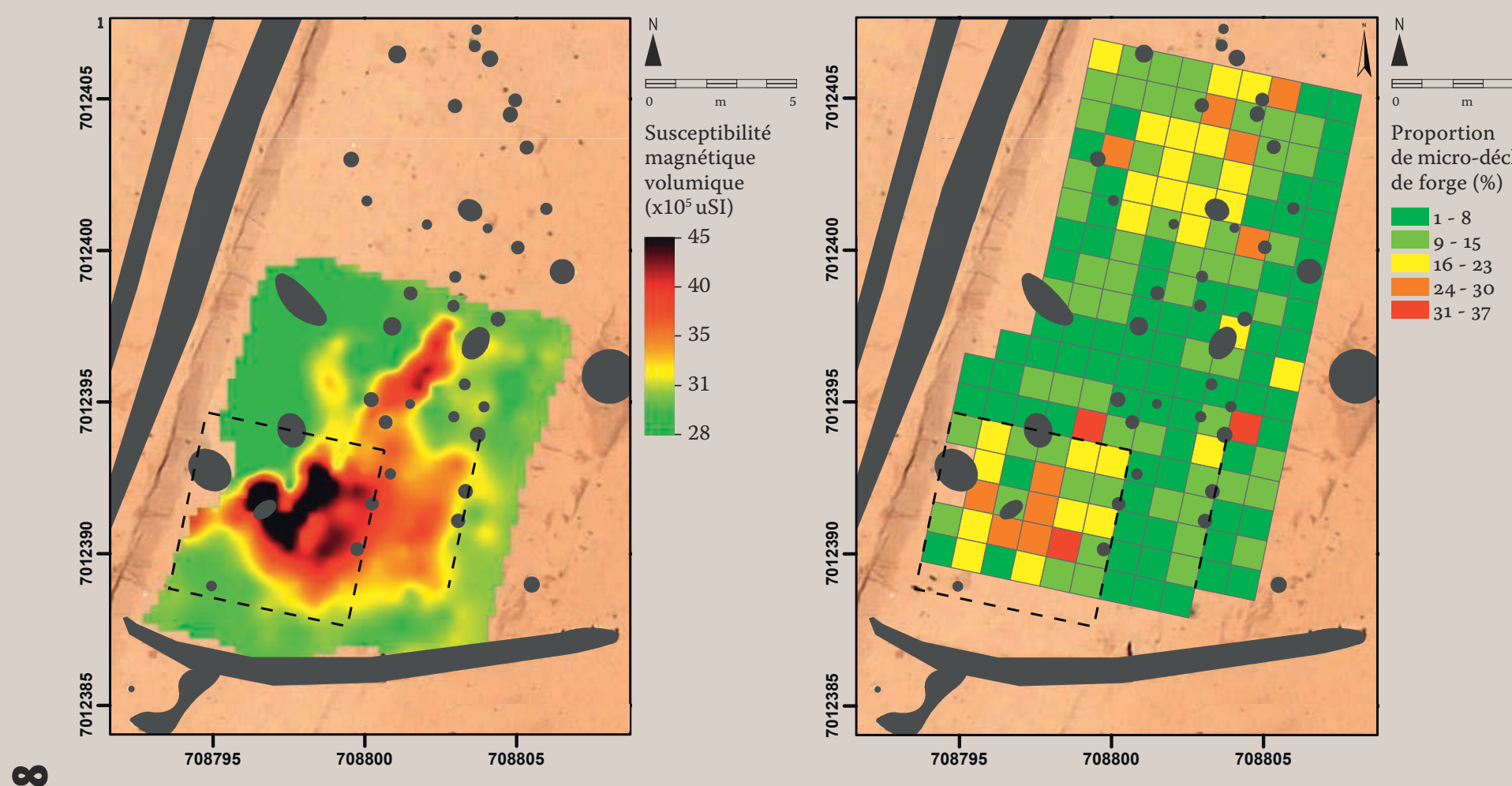

\section{Cartographie de} susceptibilité magnétique et prélèvements paléométallurgiques sur le secteur 10 du site de Sauchy-Lestrée avec proposition de zonage de la forge (en pointillés) (coord. Lambert 93).

\section{La détection d'une forge gauloise arasée} à Sauchy-Lestrée (Pas-de-Calais)

L'exemple de Sauchy-Lestrée ${ }^{1}$ est particulièrement intéressant puisque, dans ce cas précis, l'étu.de de susceptibilité magnétique a révélé la présence d’une anomalie bien structurée sur le sol naturel donc a priori stérile. Fn ce sens, il constitue certainement l'illu.stration la plu.s significative de l'apport de cette méthode pour la détection des sols de forge. À la suite du décapage, aucun indice au sol ne permettait de déceler la présence d'un espace de travail du fer. Seule la prospection géophysique a conduit à la mise en évidence d'une zone anormale qui s'est avérée en lien avec un atelier de forge fortement érodé

Des limites franches sont cependant présentes et peuvent être interprétées comme des effets de paroi qui sont à mettre en relation avec le plan archéologique. Un carroyage a été implanté sur ce secteur pour la réalisation des prélèvements. À la différence du site de Noyon, seulement 3 litres de sédiments ont été prélevés par carré. Une fois traité, l'ensemble des échantillons n'a mis en évidénce, en moyenne, qu'une faible proportion. d'éléments magnétiques par carré qui s'explique par l'état de conservation et d'arasement du site. Inn effet, les seuls déchets présents sont ceux ayant migré en profondeur notamment par les phénomènes de bioturbation. Ialgré cela, on constate une certaine corrélation entre la carte des micro-déchets et celle obtenue lors de la prospection géophysique. On note cependant que, sur certains endroits de l'espace testé, les résultats divergent fortement. In effet, certains carrés pourtant très magnétiques n'ont pas révélé de fortes proportions de microdéchets. Il faut donc envisager qu'une autre action anthropique que le forgeage du métal ait pu avoir lieu. I'hypothèse d'une anomalie liée à des phénomènes de chauffe peut être émise. Cependant, peu d'indices semblent susceptibles d'apporter des réponses à cette interrogation pour le moment. À l'emplacement où les fortes susceptibilités sont corrélées à de fortes proportions d'éléments magnétiques, l'observation microscopique a permis de détecter la présence de battitures lamellaires et en billes. Ces observations donnent une première image globale sur l'organisation de cette forge qui daterait de la première moitié du $\mathrm{I}^{\mathrm{er}}$ siècle avant notre ère (étude en cours, Lefèvre, Sarrazin, à paraître). Ainsi, l'espace où était frappé le métal semble se situer dans la partie sud-ouest de la forge dont l'une des structures en creux

découvertes dans ce secteur aurait pu servir de point d'ancrage à l'enclume. La cartographie réalisée

après le traitement des sédiments a également permis de constater un prolongement des effets de parois détectés lors de la prospection géophysique et ainsi apporter une meilleure connaissance du bâtiment abritant la forge. On observe une continuité de ce dernier vers le nord-est avec un second. espace où une concentration d'éléments magnétiques - qui n'avait pas été détectée lors de la prospection. géophysique - semble exister. Du fait du peu d'indices découverts, il est difficile d'interpréter ce second. secteur. Jist-il possible d'y voir un lieu de passage privilégié lors des différentes séquences de nettoyage de la forge? Les différents trajets entre la zone de travail et les espaces de rejet (observés en périphérie de l'espace métallurgique) auraient-ils pu provoquer un đépôt de micro-déchets magnétiques dans ce secteur? IMalheureusement, le degré d'arasement du. site laisse cette idée à l'état d'hypothèse. On voit donc pour l'exemple de Sauchy-Lestrée que, sans un travail préalable de détection géophysique, aucun indice d'une activité du travail du fer sur le site n'aurait pu être décelé. De même, il est évident que les concentrations faibles et éparses de déchets scoriacés mis au jour sur le site n'auraient pas pu, à elles seules, apporter des indices significatifs d'un tel artisanat. Fn revanche, on note que l'adéquation. des anomalies magnétiques avec la présence de battitures n'est pas parfaite dans ce cas et que d'autres phénomènes doivent être pris en compte. la direction de Philippe Lefèvre, Inrap, d'avril à septembre 2012 . 
les espaces métallurgiques mais d'apporter une caractérisation plus fine de l'activité. Pour chacun des sites étudiés, quel que soit leur état de conservation, les zones de martelage ont pu être distinguées et des hypothèses sur l'emplacement de l'enclume ont été avancées par l'analyse croisée. Cette méthode présente également l'intérêt d'optimiser à la fois les quantités prélevées (seulement trois litres de sédiments) et les moyens humains alloués sur le terrain et lors de la phase de postfouille. Enfin, ces résultats viennent compléter l'étude des déchets scoriacés retrouvés sur le site, ce qui permet de mieux cerner l'activité des différentes forges. Malgré tout, l'exemple de Sauchy-Lestrée montre que cette méthodologie nécessite d'être affinée. En effet, certaines zones de susceptibilité élevée ne semblent pas avoir été produites par la présence de micro-déchets magnétiques mais par une autre action anthropique. Il est donc intéressant de poursuivre cette démarche en tentant de mieux distinguer les anomalies générées par le travail du fer de celles produites par des zones de chauffe par exemple. Une piste de travail intéressante consiste à étudier d'autres paramètres magnétiques et plus particulièrement la viscosité magnétique.

L'aspect principal de la démarche exposée ici réside dans la mise en œuvre d'une méthode simple et rapide visant à la détection des zones dédiées au travail du fer. Une signature géophysique caractéristique, liée à la présence de micro-déchets ferreux, permet d'identifier ces zones et d'apporter des éléments de réflexion dès la phase de terrain. Cette approche offre la possibilité de reconsidérer l'organisation même du lieu dédié au travail du fer en mettant en avant des zones n'ayant laissé aucune trace visible mais qui, au vu des caractéristiques physiques (forte susceptibilité magnétique due à des battitures, zone de chauffe) et de l'organisation spatiale (présence d'effets de parois), ne peuvent être ignorées dans l'interprétation finale.
Références bibliographiques

AitKen M.J., 1958, « Magnetic prospecting », Archaeometry, 1, p. 24-29.

Benech C., Marmet E., 1999, « Optimum depth of investigation and conductivity response rejection of the different electromagnetic devices measuring apparent magnetic susceptibility », Archaeological Prospection, 6, 1, p. 31-45.

BEST C., MARMET E., à paraitre, «Étude paléométallurgique sur le site de Beaune-la-Rolande », in CrIBELLIER C., Rapport de fouille sur le site I2/2-3 (A19) de Beaunela-Rolande, «la Justice » et «les Guénines ».

Bonnamour G., Florsch N., TéreygeOl F., 2009, «Les prospections des ferriers de Castel-Minier : approche interdisciplinaire », ArchéoSciences. Revue d'archéométrie, 31, p. 37-44.

Саввої S., Dunikowski C., 1995, La sidérurgie chez les Sénons : Les ateliers celtiques et gallo-romains des Clérimois (Yonne), Maison des Sciences de l'Homme (coll. DAF, 51), $183 \mathrm{p}$.

Crew P., 2002, « Magnetic mapping and dating of prehistoric and medieval iron-working sites in northwest Wales ", Archaeological Prospection, 9, 3, p. 163-182.

Dabas M., Dieudonné-Glad N., Poirier P., 2002, «Caractérisation des structures d'une forge antique: approche archéologique, géophysique et anthracologique », Revue d'archéométrie, 26, p. 141-154

De Muylder M. et al., à paraître, Canal Seine-Nord Europe, fouille 34, Picardie, Oise, Noyon, « La Mare aux Canards ». "Une villa aristocratique de la cité des Viromanduens », rapport final d'opération, Inrap-SRA Picardie.

Gustafsson N.B., Viberg A., 2012, « Tracing Hightemperature Crafts: Magnetometry on the Island of Gotland, Sweden », Archaeological Prospection, 19,3 , p. 201-208.

Henton A., à paraître, Saultain «Rue H. Barbusse » rapport final d'opération, Inrap-SRA Nord-Pas-de-Calais

Hulin G., Prilaux G., Talon M., 2014, « Intégration de la géophysique à un projet archéologique d'envergure : l'exemple du projet canal Seine-Nord Europe », Revue Archéologique de Picardie, 1/2, p. 245-26o.

LAMOTTE D. et al., à paraître, Canal Seine-Nord Europe, fouille 25, Picardie, Somme, Eterpigny-Barleux. «Les fouilles archéologiques sur la plate-forme agro-industrielle de Péronne dans le cadre de la construction du Canal Seine-Nord Europe », rapport final d'opération, Inrap-SRA Picardie.

LEBLANC J.-C., 2001, «Archéométrie des battitures de forge. Lidentification des travaux de post-réduction de l'épuration au traitement/fonctionnel de l'objet », in L'obtenció del ferro pel procediment directe entre els segles IV $i$ XIX, Andorra la Vella: Govern d'Andorra, Ministeri de cultura, p. 367-38o.

LE Borgne E., 1955, "Susceptibilité magnétique anormale du sol superficiel », Annales de géophysique, 11, p. 399-419.

LE BorGNE E., 1965, « Les propriétés magnétiques du sol. Application à la prospection des sites archéologiques », Archaeo-physika, 1, p. 1-21.

LeFÈVRE P., SARRAZIN S., à paraitre, Canal Seine-Nord Europe, fouille 32, Nord-Pas-de-Calais, Pas-de-Calais, Marquion/Sauchy-Lestrée. «VolumeIV:les occupations domestiques protohistoriques », in PRILAUX G., La plate-forme multimodale de Marquion/SauchyLestrée (62), du néolithique au Bas-Empire, rapport final d'opération, Inrap-Nord-Pas-de-Calais.

Mangin M., 2004, Le fer, Paris, Errance (collection Archéologie), $239 \mathrm{p}$.

MARMET E., 2000, Cartographie à large maille de la susceptibilité magnétique du sol pour une évaluation archéologique sur les grands tracés, thèse de doctorat, Université Pierre et Marie Curie, Paris, $236 \mathrm{p}$.

Mullins C.E., 1974, « The magnetic properties of the soil and their application to archaeological prospecting », Archaeo-physika, 5, p. 143-147.

Tite M.S., Mullins C.E., 1971, « Enhancement of the magnetic susceptibility of soils on archaeological sites ", Archaeometry, 13, p. 209-219.

VERNON R.W., MCDONNELl G., SCHMidt A., 1998 « The geophysical evaluation of an iron-working complex: Rievaulx and environs, North Yorkshire », Archaeological Prospection, 5, 4, p. 181-201. 